\title{
ERK1/2 pathway regulates coxsackie and adenovirus receptor expression in mouse cardiac stem cells
}

\author{
JINGJIN LIU ${ }^{1,2}$, QIANG SUN ${ }^{3}$, YONGSHUN WANG ${ }^{1,2}$, JINJIN CUI ${ }^{1,2}$, MAOMAO ZHANG ${ }^{1,2}$, \\ LILI LI $^{1}$, HAIBO JIA ${ }^{1}$, LULU ZHANG ${ }^{1}$, BIN ZHU ${ }^{1}$, MIAOMIAO JIANG ${ }^{1}$, \\ BO $\mathrm{YU}^{1,2}$ and SHUO ZHANG ${ }^{1}$
}

\author{
${ }^{1}$ Department of Cardiology, The Second Affiliated Hospital of Harbin Medical University, Harbin, Heilongjiang 150086; \\ ${ }^{2}$ Key Laboratories of Education, Ministry for Myocardial Ischemia Mechanism and Treatment (Harbin Medical University), \\ Ministry of Education, Harbin, Heilongjiang 150086; \\ ${ }^{3}$ Department of Cardiology, The First Hospital of Qinhuangdao, Qinhuangdao, Hebei 066000, P.R. China
}

Received July 22, 2015; Accepted October 10, 2016

DOI: $10.3892 /$ etm.2017.4414

\begin{abstract}
Cardiac stem cells (CSCs) are the most promising and effective candidates for the therapy of cardiac regenerative diseases; however, they have marked limitations. For instance, the implantation of CSCs is hampered by factors such as their sustainability and long-term durability. Gene modification appears to be the most effective method of optimizing CSCs and gene therapy trials have demonstrated that efficient gene transfer is key to achieving therapeutic efficacy. However, the transduction ability of adenovirus (Ad) is limited. Previous studies have reported that low expression of coxsackie and adenovirus receptor (CAR) in target cells decreases the transduction efficiency. A promising method for improving Ad-mediated gene transfer is to increase CAR expression in target cells. The present study investigated the effect of the Raf-mitogen-associated protein kinase (MAPK) kinase (MEK)-extracellular signal-associated protein kinase (ERK) signaling pathway on the expression of CAR on CSCs, as this pathway decreases cell-cell adhesion via cell surface molecules. The results demonstrated that interference with the Raf-MEK-ERK signaling pathway by knockdown of ERK1/2 upregulated the expression of CAR. The entry of the Ad into the cells was increased following inhibition of ERK1/2. Moreover, following knockdown of CAR, the entry of Ad into cells was decreased. However, knockdown of c-Jun N-terminal kinase and p38 as other components of the MAPK pathway did not affect CAR expression. Therefore, CAR expression in CSCs may be mediated via the Raf-MEK-ERK signaling
\end{abstract}

Correspondence to: Professor Shuo Zhang, Department of Cardiology, The Second Affiliated Hospital of Harbin Medical University, 246 Xuefu Road, Harbin, Heilongjiang 150086, P.R. China

E-mail: shuozhang39@163.com

Key words: coxsackie and adenovirus receptor, gene therapy, extracellular signal-regulated kinase $1 / 2$, cardiac stem cells pathway. Upregulation of CAR by knockdown of ERK1/2 may significantly improve Ad-mediated genetic modification of CSCs in the treatment of cardiovascular diseases.

\section{Introduction}

The efficacy of cardiac stem cell (CSC) transplantation for cardiomyoplasty is limited due to poor survival of the implanted cells in ischemic hearts $(1,2)$. Furthermore, strategies aiming to improve the efficiency of CSC transplantation have shown little success.

Genetically modified or re-constructed adenoviral vectors have been widely used to optimize CSCs for cellular cardiomyoplasty in the treatment of cardiovascular disease (3-7). McCormick (8) reported that improved adenoviruses effectively deliver therapeutic genes or drugs into target cells. Recombinant adenoviral vectors have been constructed, which have shown promising effects in clinical and pre-clinical studies (9). Furthermore, the efficiency of gene therapy was found to mainly be based on the binding ability of the internalized adenovirus and its target.

The coxsackie and adenovirus receptor (CAR) is a transmembrane protein with a molecular weight of $46-\mathrm{kDa}$. CAR interacts with tight junction protein complexes in normal human cells (10). It has also been shown to enhance the viral attachment capability by interacting with the adenovirus fiber-knob protein in cells (11). The loss or decrease in expression of CAR may significantly decrease viral infectivity, however, this decrease in viral infectivity may be rescued by ectopic expression of CAR protein (12). It has therefore been suggested that the amount and location of CAR expression may determine viral infectivity. Internalization of adenoviruses in cells is primarily mediated by binding of the virus penton base to $\alpha v \beta 3$ - and $\alpha v \beta 5$-integrins (13). Studies have also indicated that decreased expression of CAR is observed in highly malignant tumors (14-16). Molecular cues and signaling pathways modulating CAR expression in CSCs have remained elusive. While certain studies have investigated the application of CAR in adenoviral vector-mediated expression for the regeneration of transplanted CSCs, the potential molecular mechanisms 
of the regulation of CAR expression remain to be fully elucidated. The expression of tight junction proteins is mediated by triggering the oncogenic signaling pathway, for instance, by triggering the Ras-activated extracellular signal-regulated kinase (ERK) signaling pathway in cells $(16,17)$. The Raf-mitogen-activated protein kinase (MAPK) kinase (MEK) -ERK signaling pathway may serve a central role in regulating CAR and viral uptake, by which adenoviral vector therapy may be optimized $(18,19)$. The present study demonstrated that vector-mediated inhibition of the Raf-MEK-ERK pathway enhanced CAR expression in mouse CSCs, therefore providing potential targets for improving adenovirus-based treatments. The results indicated that knockdown of ERK1/2 expression led to an upregulation of CAR on the CSC surface, which may be associated with increased adenoviral uptake into cells.

\section{Materials and methods}

Cell lines and CSC isolation and culture. In the present study, human endometrial cancer cells from the ishikawa (ISK) cell line were donated by the Second Affiliated Hospital of Harbin Medical University (Harbin, China). CSCs were isolated from the extracted hearts of Balb/c mice (18-25 g) using a method previously published by Beltrami et al (20), Liu et al $(21,22)$ and Wang et al (23). All Balb/c mice were purchased from the Department of Laboratory Animal Science of the Second Affiliated Hospital of Harbin Medical University (Harbin, China). In brief, heparin $(5,000 \mathrm{IU} / \mathrm{kg})$ was intraperitoneally injected into the mice $20 \mathrm{~min}$ prior to the experiments. All mice were subsequently sacrificed by cervical dislocation. The heart was excised and the aorta was rapidly cannulated. Subsequently, the cannulated hearts were mounted and observed using a Langendorff perfusion system (Hugo Sachs Elektronik-Harvard Apparatus GmbH, March, Germany) with constant flow. The perfusion pressure of the hearts was observed and monitored. In order to remove the blood from the mouse hearts, they were treated and perfused with $\mathrm{Ca}^{2+}$-free Tyrode's solution (BSS-350; Boston BioProducts, Ashland, MA, USA) for 5-10 min. Subsequently, the hearts were digested with collagenase $(0.5 \mathrm{mg} / \mathrm{ml}$; Sigma-Aldrich; Merck Millipore, Darmstadt, Germany) and trypsin $(0.5 \mathrm{mg} / \mathrm{ml}$; Gibco; Thermo Fisher Scientific, Inc., Waltham, MA, USA) for $30 \mathrm{~min}$ at $37^{\circ} \mathrm{C}$. The heart tissue was subsequently extracted and cleaved, and the cell suspension was filtered with a prepared Steriflip instrument (SCNY00100-1EA; EMD Millipore, Billerica, MA, USA). The obtained suspension was treated with fluorescein isothiocyanate (FITC)-labeled rat anti-mouse CD117/c-kit polyclonal antibody (1:100, catalog no. 553354, BD Biosciences, Franklin Lakes, NJ, USA) and the suspension was separated using MACS anti-FITC microbeads (Miltenyi Biotech, Bergisch Gladbach, Germany). Small round cells, containing most of the c-kit ${ }^{+}$sub-population, were harvested. The small round cells were cultured in HyClone Dulbecco's Modified Eagle's medium (DMEM)/F12 (Thermo Fisher Scientific, Inc.) supplemented with $10 \mathrm{ng} / \mathrm{ml}$ bovine fibroblast growth factor (PeproTech, Rocky Hill, NJ, USA), $10 \%$ fetal bovine serum (FBS, Hyclone; GE Healthcare Life Sciences, Logan, UT, USA), epidermal growth factor (PeproTech), $10 \mathrm{ng} / \mathrm{ml}$ insulin growth factor (PeproTech) and $10 \mathrm{ng} / \mathrm{ml}$ leukemia inhibitory factor (Sigma-Aldrich; Merck
Millipore) for 3-5 days at $37^{\circ} \mathrm{C}$. The cells were subjected to subsequent examinations or experiments whenever they were recovered. The experimental animal and cell experimental processes and protocols of the present study were approved by the Ethics Committee of Harbin Medical University (Harbin, China).

Small interfering RNA (siRNA) silencing. Gene silencing by siRNA involves a small double-stranded RNA that degrades target mRNA. The siRNA duplex was synthesized at Shanghai GenePharma Co., Ltd (Shanghai, China). To detect the regulatory roles of MAPKs in mediating CAR expression, additional CSCs were transfected with 100 pmol siRNA against; c-Jun N-terminal kinase (JNK), forward, 5'-UCAAGGAAUAGU GUGUGCAGCUUAU-3', and reverse 5'-CAGCCCAGT AATATAGTAGTA-3'; p38 forward, 5'-GGACCUCCUUAU AGACGAAUU-3' and reverse, 5'-GGACCUCCUUAUAGA CGAAUU-3'; ERK1/2 forward, 5'-GACCGGAUGUUAACC UUUAUU-3' and reverse, 5'-GACCGGAUGUUAACCUUU AUU-3'; CAR forward, 5'-GACGCAUCUAUAAAUGUG AUU-3' and reverse, 5'-GACGCAUCUAUAAAUGUGAUU-3', using Lipofectamine ${ }^{\circledR}$ RNAiMAX (Invitrogen; Thermo Fisher Scientific, Inc.) for $48 \mathrm{~h}$. In the control group, cells were treated with either a transfection reagent (vehicle) or a non-targeting siRNA (siRNA-NC), forward, 5'-UUCUCCGAACGUGUC ACG-3' and reverse, 5'-ACGUGACACGUUCGGAGAATT-3' (Shanghai GenePharma Co., Ltd.).

In vitro adenoviral vector transduction. Recombinant adenovirus-green fluorescence protein (Ad-GFP) was purchased from Shanghai GenePharma Co., Ltd. Ad transductions were performed in six-well plates, with cells seeded at $2-5 \times 10^{5} /$ well $48 \mathrm{~h}$ previously. Virus particles $\left(10^{\%} /\right.$ well $)$ were diluted in serum-free DMEM/F12 (1 ml/well; Thermo Fisher Scientific, Inc.) and then incubated at $37^{\circ} \mathrm{C}$ with the cells for $90 \mathrm{~min}$. Subsequently, the viral suspension was replaced with complete culture medium (containing FBS). Following 72 h transduction, cells were washed with phosphate-buffered saline (PBS) and subjected to flow cytometric and fluorescence microscopic analysis.

Immunofluorescence. The cells were incubated with $4 \%(\mathrm{v} / \mathrm{v})$ formaldehyde for $15 \mathrm{~min}$ at room temperature. Subsequently, the cells were washed three times with PBS for 5 min each and blocked with $10 \%$ bovine serum albumin (HyClone; GE Healthcare Life Sciences). The cells were then incubated with rabbit anti-CAR antibody (1:100 dilution; cat. no. H-300; Santa Cruz Biotechnology, Inc., Dallas, TX, USA) for $1 \mathrm{~h}$ at $37^{\circ} \mathrm{C}$. The cells were washed again three times with PBS ( 5 min each) and treated with Alexa Fluor 555-labeled goat anti-rabbit antibody (1:1,000 dilution; cat. no., A-21428, Invitrogen; Thermo Fisher Scientific, Inc.) at $37^{\circ} \mathrm{C}$ in the dark for $1 \mathrm{~h}$. Subsequent to washing with PBS, the nuclei were counter-stained with the 4', 6-diamidino-2-phenylindole (Sigma-Aldrich; Merck Millipore). Finally, the cells were observed and examined under a fluorescence microscope (DMI4000B; Leica Microsystems, Wetzlar, Germany).

Western blot analysis. The cells were lysed following hypertrophy induction using buffer radioimmunopreciptation assay 
buffer (Abcam, Cambridge, MA, USA) by adding a protein inhibitor cocktail (Roche Diagnostics, Indianapolis, IN, USA) to recover the extract. To separate proteins, samples were denatured at $97.5^{\circ} \mathrm{C}$ for $>2 \mathrm{~min}$. A total of $20 \mu \mathrm{g}$ of protein was resolved by SDS-PAGE at $12.5 \%$ and transferred onto polyvinylidene difluoride membranes (EMD Millipore), which were then blocked with 5\% non-fat milk in Tris-buffered saline containing Tween 20 (TBST) for $1 \mathrm{~h}$ at room temperature. Mouse anti-phosphorylated (p)-JNK (1:1,000; CST\#9255), anti-p-p38 (1:1,000; CST\#9216), anti-p-ERK1/2 (1:1,000; CST\#9106), anti-total (t)-JNK (1:1,000; CST\#3708), anti-t-p38 (1:1,000; CST\#9217) and anti-t-ERK1/2 (1:1,000; CST\#4696) were all purchased from Cell Signaling Technology, Inc. (Danvers, MA, USA). Rabbit anti- $\beta$-actin (1:200; PR-0255) was purchased from Zhongshan Goldenbridge Biotechnology Co. Ltd, Beijing, China and mouse anti-CAR monoclonal antibody (1:200; sc-373791) was purchased from Santa Cruz Biotechnology, Inc. The membranes were incubated with the above antibodies overnight at $4^{\circ} \mathrm{C}$. Subsequently, the membranes were incubated with horseradish peroxidase (HRP)-conjugated Affinipure goat anti-rabbit immunoglobulin (Ig) G (H+L) polyclonal antibody (1:2,000; ab6721, Abcam) and HRP-conjugated rabbit anti-mouse $\operatorname{IgG}(\mathrm{H}+\mathrm{L})$-labeled polyclonal antibody (HRP; 1:2,000; ab6728, Abcam) at $37^{\circ} \mathrm{C}$ $1 \mathrm{~h}$ and washed with TBST solution. Bands were then visualized by enhanced chemiluminescence (ECL) detection using BeyoECL Plus (Beyotime Institute of Biotechnology, Inc., Haimen, China). Images of the gels were captured on X-ray film, following which the bands were stripped with $5 \mathrm{ml}$ stripping buffer (CoWin Biotech, Beijing, China) at room temperature for $15 \mathrm{~min}$ and hybridized with $\beta$-actin (1:200; TA890010, OriGene Technologies, Inc., Beijing, China) at room temperature for $15 \mathrm{~min}$ for normalization. Protein bands were quantified by densitometry using the Tanon Gel Imaging System (Shanghai Tanon Co. Ltd., Shanghai, China).

Quantitative reverse transcription quantitative polymerase chain reaction $(R T-q P C R)$. Total RNA was isolated from the cells subjected to the different experimental conditions with TRIzol (Invitrogen; Thermo Fisher Scientific, Inc.), according to the manufacturer's instructions. Following pretreatment with RNase-free DNase I, 2- $\mu \mathrm{g}$ miRNA was subjected to the protocols of the miScript Reverse Transcription kit and the miScript SYBR Green PCR kit (Qiagen Benelux B.V, Venlo, the Netherlands). RNA was converted into cDNA by reverse transcription with the Transcriptor First Strand cDNA Synthesis kit (Invitrogen; Thermo Fisher Scientific, Inc.) qPCR was performed using a FastStart Universal SYBR Green Master Mix (ROX) (Roche Applied Science, Mannheim, Germany) in a $10 \mu \mathrm{l}$ reaction volume on an ABI 7900 Genome Analyzer System (5 $\mu \mathrm{l} \mathrm{SYBR}$ green Mix, $200 \mu \mathrm{M}$ forward and reverse primers, $1 \mu \mathrm{l}$ cDNA template) The primer sequences are presented in Table I. The PCR reaction conditions were initial denaturation at $94^{\circ} \mathrm{C}$ for $5 \mathrm{~min}$; degeneration at $94^{\circ} \mathrm{C}$ for $15 \mathrm{sec}$, annealing at $55^{\circ} \mathrm{C}$ for $30 \mathrm{sec}$ and $72^{\circ} \mathrm{C}$ for $30 \mathrm{sec}$, for a total of 35 cycles; followed by extension at $72^{\circ} \mathrm{C}$ for 5 min. Gene amplification was confirmed after calculating the melting temperatures ( $\mathrm{Tm}$ ) for the products from the melting peak curve ( $2 \mathrm{dF} / \mathrm{dT}$ vs. temperature). All amplicons were collected and $10 \mathrm{ml}$ of each reaction was confirmed by $1.5 \%$ agarose gel electrophoresis and sequencing. A cross-point vs. logarithmic concentration standard curve was generated using serial dilutions of one of the cDNA samples or known concentrations of plasmid DNA. Negative controls were included, using cDNAs synthesized in the same manner as described above, but without reverse transcriptase. Each cDNA sample was run in triplicate. Relative mRNA expression levels were calculated using the $-2^{\Delta \Delta C q}$ method (24) based on the threshold cycle $(\mathrm{Cq})$ values and were normalized to the internal control of GAPDH. The primers (GenePharma Co., Ltd.) utilized in the present study are listed in Table I.

Flow cytometric analysis. The Ad suspension was transfected for $24 \mathrm{~h}$ and cells were subsequently cultured for $48 \mathrm{~h}$ and harvested. Then cells were re-suspended at a density of $1 \times 10^{6}$ cells in $500 \mu \mathrm{l}$ binding buffer. A total of 10,000 events were collected and the proportion of GFP cells was analyzed with a FACSCalibur flow cytometer (BD Biosciences).

Statistical analysis. Statistical analysis was performed using SPSS 18.0 software (SPSS Inc., Chicago, IL, USA). Values are expressed as the mean \pm standard error. Comparisons between the parameters were analyzed using Student's $t$-test. $\mathrm{P}<0.05$ was considered to indicate a statistically significant difference.

\section{Results}

Characterization of CAR expression on $m C S C s$. In a preliminary study by our group, immunofluorescent microscopic analysis only demonstrated small amounts of CAR expression at the surface of SW480 cells. In the present study, CAR protein was also located in the cell cytoplasm, while small amounts were located at the cell surface, which may have posed a disadvantage regarding Ad uptake in mCSC and ISK cells (Fig. 1).

MAPK pathway silencing in $m C S C s$. In order to further explore the influence of the MAPK pathway on CAR expression in mCSCs, ERK1/2, JNK and P38 were individually silenced with siRNA (Fig. 2). Following 48 h transfection, a knockdown efficiency of $>80 \%$ of the targeted proteins was observed in the cells transfected with specific siRNAs against ERK1/2, JNK and p38 (Fig. 2), which was also sustained for up to $72 \mathrm{~h}$ (data not shown).

Knockdown of ERK1/2 increases expression of CAR protein and its abundance at the surface of $m C S C s$. Following the silencing of ERK1/2 with siRNA, mCSCs exhibited significantly increased CAR protein expression, while there was no such effect in siRNA-NC (Fig. 3A). Immunofluorescence microscopy was also performed to investigate whether inhibition of Raf-MEK-ERK signaling affected not only CAR expression but also its sub-cellular localization. In control cells, CAR was only weakly expressed in the cytoplasm and CAR expression at the cell surface was low. However, after ERK1/2 silencing with siRNA-ERK1/2, this expression pattern clearly changed, with CAR being mainly located at the cell surface at sites of cell-cell contact (Fig. 3B). Furthermore, results from RT-qPCR indicated that in mCSCs, CAR mRNA levels were significantly increased following the silencing of ERK1/2 with 
siRNA (Fig. 3C). These results showed that the changes of CAR levels are specifically associated with signaling through the ERK1/2 pathway.

Furthermore, the present study investigated the effects of JNK and p38 silencing on CAR expression. As well as ERK1/2, the JNK and p38 pathways are the other two major MAPK signal transduction pathways. The results indicated that transfection of JNK- and P38-siRNA had no effect on CAR protein expression (Fig. 3A). In order to evaluate whether the CAR protein changes were caused by changes in CAR gene transcription, RT-qPCR was performed. In mCSCs, CAR mRNA levels were unchanged after silencing of JNK or p38 with siRNA (Fig. 3C). The above results suggested that the ERK1/2 but not the JNK and p38 signaling transduction pathways affect CAR expression. However, these effects require investigation in other cell types.

ERK1/2 silencing increases Ad uptake in $\mathrm{mCSCs}$, which is inhibited by CAR siRNA. As CAR acts as the primary cellular binding site for the Ad (19), the present study hypothesized that increased CAR expression at the cardiac stem cell surface may lead to enhanced viral uptake. In order to verify this hypothesis, CSCs were first infected with a non-replicating Ad-GFP following treatment with ERK1/2-, JNK- or P38-siRNA. The FACS assay showed significantly increased infectivity of CSCs treated with the ERK1/2-siRNA compared with control cells. However, treatment with JNK- and P38-siRNA did not affect the infectivity of CSCs (Fig. 4A).

To evaluate whether ERK1/2-siRNA increases the Ad transgene expression in $\mathrm{mCSCs}$, fluorescence microscopy was used. The results showed that in the ERK1/2-siRNA transfected mCSCs, GFP expression was markedly increased (Fig. 4B).

To further test the hypothesis that the Ad uptake is improved by upregulation of CAR, mCSCs were transfected with CAR siRNA. Western blot analysis revealed that after $48 \mathrm{~h}$ transfection, siRNA-CAR had a $>80 \%$ knockdown efficiency (Fig. 5A), which was sustained for up to $72 \mathrm{~h}$ (data not shown). Following treatment with CAR siRNA, the infectivity of CSCs by Ad-GFP was decreased and remained at low levels when ERK1/2 siRNA was co-transfected (Fig. 5B). These results indicated that decreases in CAR expression at the cardiac stem cell surface inhibited viral uptake and that the presence of CAR is required for Ad uptake, which may be increased via inhibition of the ERK1/2 pathway, rather than being affected by the other two branches of the MAPK pathway.

\section{Discussion}

The present study aimed to explore the effects of ERK1/2 signaling on CAR expression in $\mathrm{mCSCs}$ as a potential target for Ad-based therapies. The main findings obtained were the following: i) CAR protein is located in the cell cytoplasm, while a small portion of the protein is located at the surface of mCSCs and ISK cells; ii) knockdown of ERK1/2 increases the expression of CAR protein in mCSCs but not JNK and P38; iii) silencing of ERK1/2 leads to upregulation of CAR protein at the cell surface in mCSCs, while silencing of JNK and P38 does not; and iv) ERK1/2 siRNA increases Ad uptake in mCSCs, which is abrogated by co-transfection with CAR
Table I. Primers for polymerase chain reaction.

\begin{tabular}{ll}
\hline Gene & \multicolumn{1}{c}{ Primer sequences } \\
\hline CAR & F: 5'-GCATCACTACACCCGAACAGA-3'; \\
& R: 5'-CAAGAACGGTCAGCAGGAAT-3'; \\
GAPDH & F: 5'-GGCACAGTCAAGGCTGAGAATG-3'; \\
& R: 5'-ATGGTGGTGAAG ACG CCA GTA-3';
\end{tabular}

CAR, coxsackie and adenovirus receptor; F, forward; R, reverse.
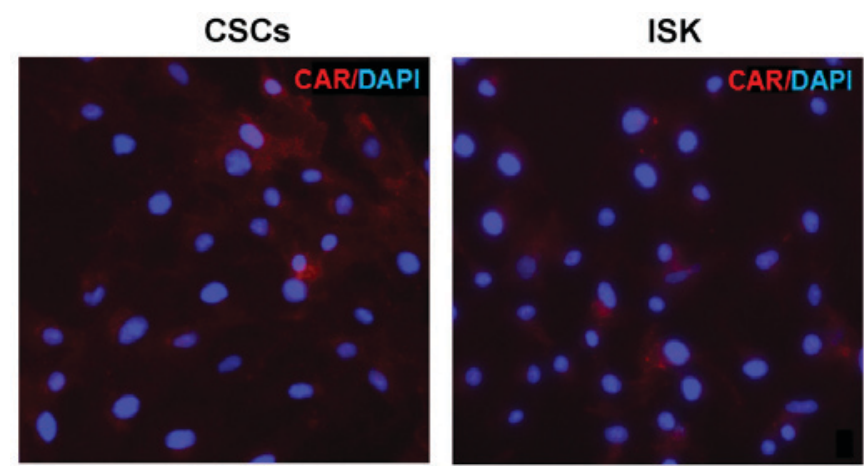

Figure 1. Characteristics of CAR expression and inhibition of mitogen-activated protein kinase on murine CSCs. Immunofluorescence analysis of CAR expression on CSCs and ISK cells. The nuclei were counter-stained with 4',6-diamidino-2-phenylindole (original magnification, $\mathrm{x} 400$ ). ISK, ishikawa cells; CSC, cardiac stem cells; CAR, coxsackie and adenovirus receptor.

siRNA. Overall, the results showed that in mCSCs, inhibition of ERK1/2 phosphorylation with siRNA led to elevated CAR expression, which increased Ad uptake. However, other branches of the MAPK pathway (JNK and P38) had no effect on CAR expression and Ad uptake in mCSCs.

In a number of cases, the low expression of CAR in target cells limits the efficacy of gene therapy approaches. A previous study demonstrated that the CAR receptor acts as the key factor for sufficient viral uptake (25). In normal cells, CAR is an adhesion molecule that interacts with the adherens and tight junction proteins (10). Loss of CAR on the cell surface significantly decreases viral infectivity, which may be reversed by the ectopic CAR expression. The present study focused on the regulatory mechanisms of CAR expression at the molecular level in order to explore how it modulates viral infectivity.

Firstly, CAR expression in mCSCs and the ISK cell line was detected by immunofluorescence. The findings were consistent with those of a previous study, showing that CAR protein is located in the cell cytoplasm while a small amount is present at the cell surface, which poses a disadvantage regarding Ad uptake (18).

The Raf-MEK-ERK signaling pathway downregulates cell-cell adhesion complexes. For instance, tight- and adherens junctions (17) and CAR act as cell adhesion molecules, which also participate in the formation of the apical junction complex and the tight junction $(10,26-28)$. In the present study, it was therefore hypothesized that the Raf-MEK-ERK signaling pathway contributes to the downregulation of CAR in cardiac 

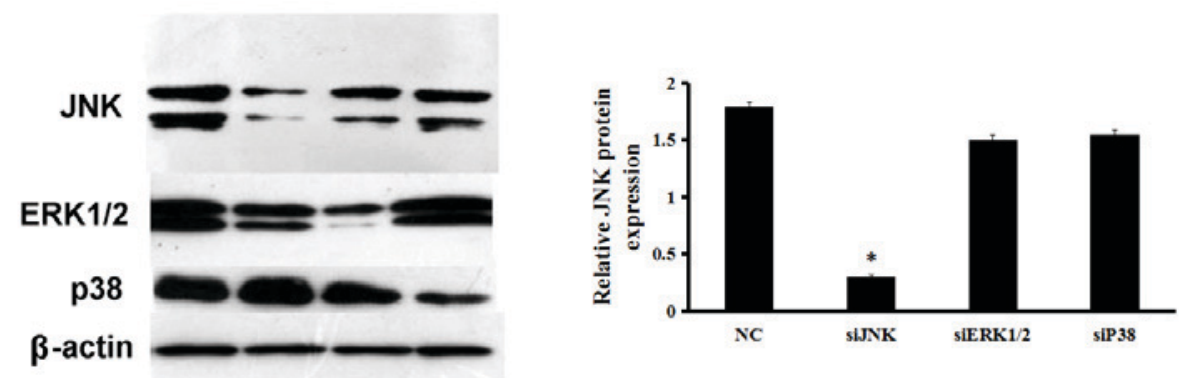

NC JNK ERK p38(siRNA)
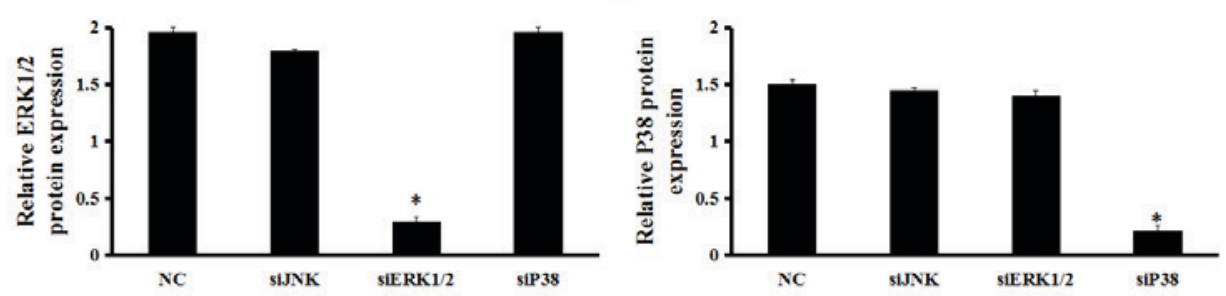

Figure 2. Cellular protein was collected after individual transfection with non-targeting, JNK, ERK1/2 or p38 siRNAs for $48 \mathrm{~h}$, followed by detection of ERK1/2, JNK and p38 protein expression by western blot analysis. "P<0.05 vs. NC. ISK, ishikawa cells; CSC, cardiac stem cell; JNK, c-Jun N-terminal kinase; ERK, extracellular signal-regulated kinase; siRNA, small interfering RNA; NC, negative control.

A
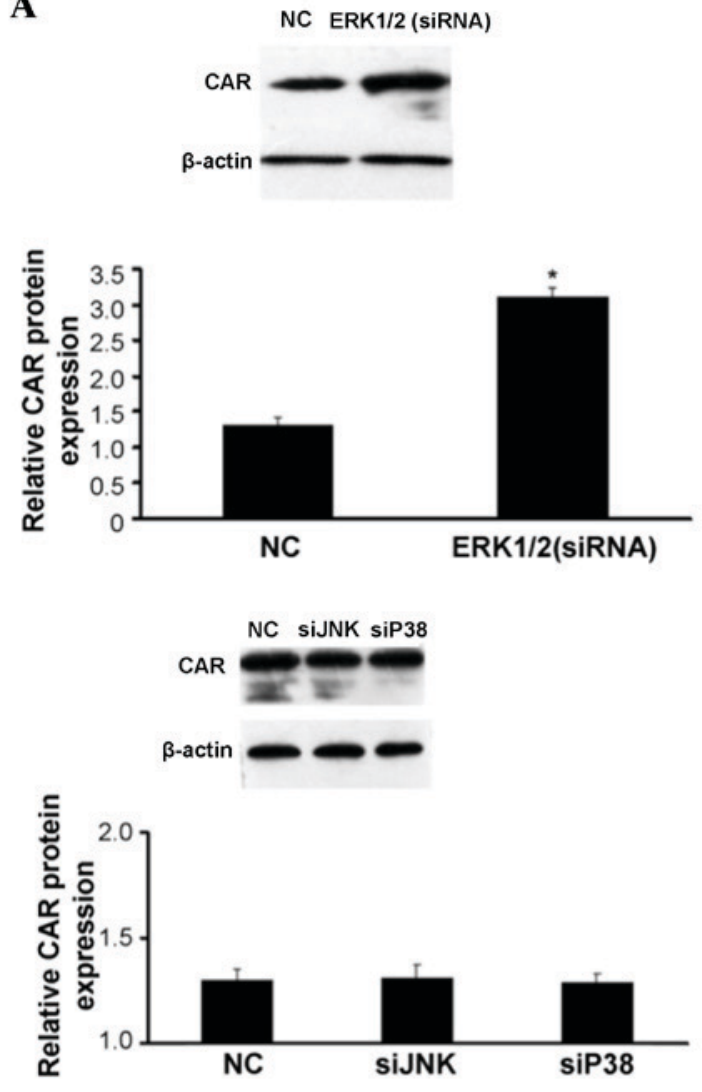

B
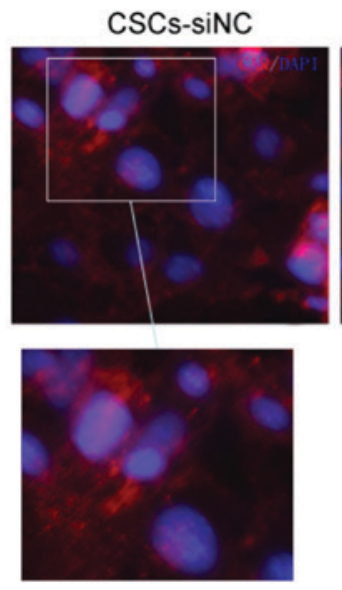

C
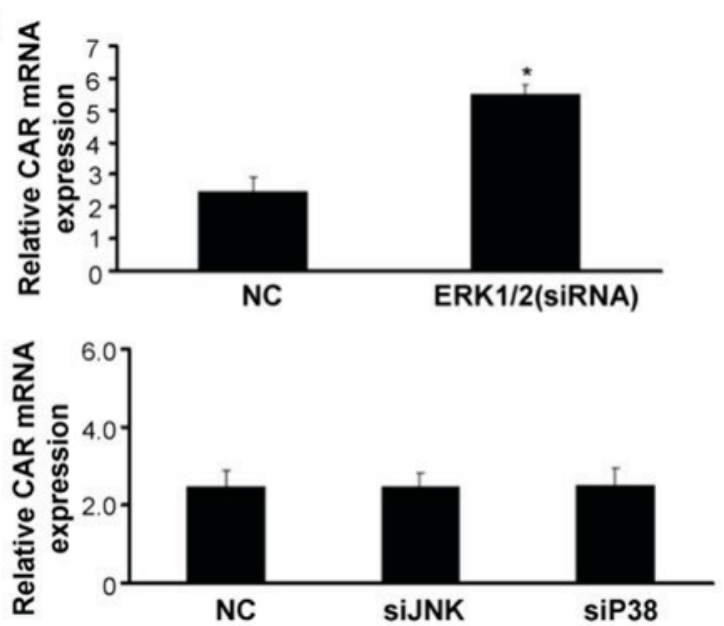

Figure 3. Effects of mitogen-activated protein kinase silencing on CAR expression in mCSCs. mCSCs were individually treated with siRNA-ERK1/2, -JNK, -P38 or -NC (control) for $48 \mathrm{~h}$. (A) Western blot analysis of CAR protein expression. Representative immunoblots and densitometrically quantified CAR protein levels. (B) Effect of ERK1/2 silencing on the subcellular CAR protein distribution observed by immunofluorescence microscopy. DNA was visualized by 4',6-diamidino-2-phenylindole staining. (C) CAR mRNA expression determined by reverse-transcription quantitative polymerase chain reaction with normalization to GAPDH. The bar graphs represent the mean values of triplicate measurements \pm standard error of the mean. ${ }^{*} \mathrm{P}<0.05$ vs. NC. mCSC, murine cardiac stem cell; JNK, c-Jun N-terminal kinase; ERK, extracellular signal-regulated kinase; siRNA, small interfering RNA; CAR, coxsackie and adenovirus receptor; $\mathrm{NC}$, negative control. 


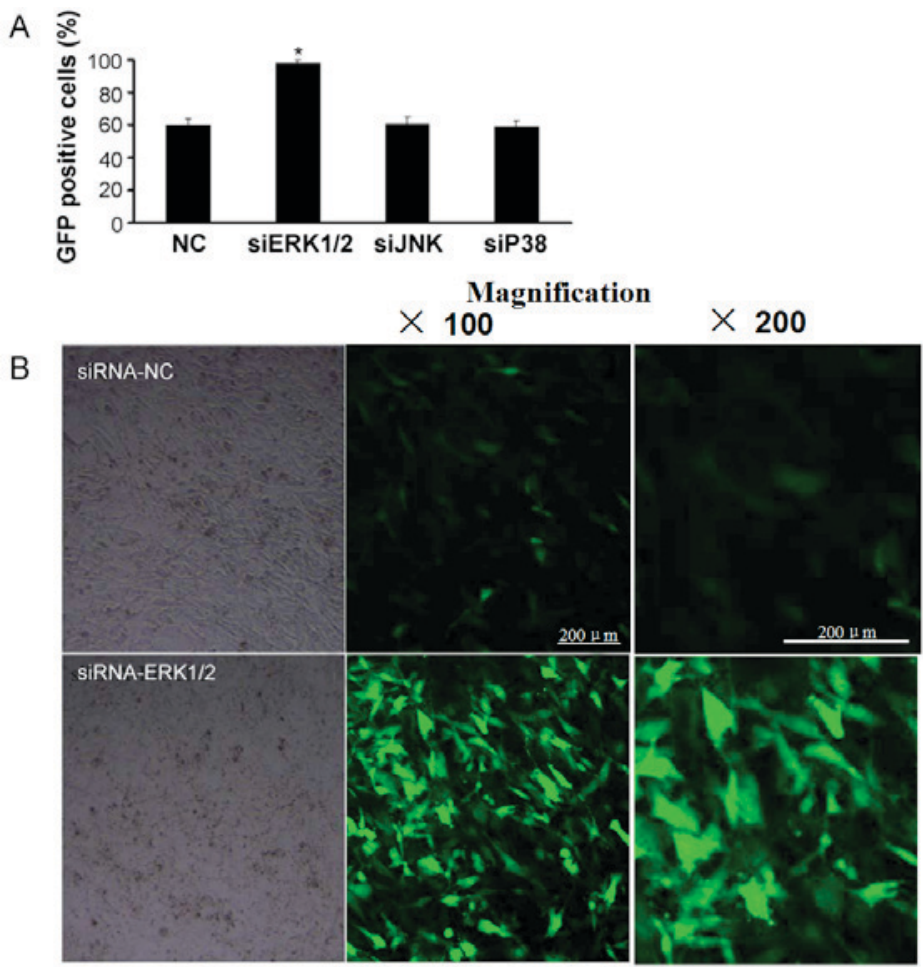

Figure 4. Changes in viral uptake following ERK1/2 silencing. The infectivity of a GFP-expressing, non-replicating adenovirus at a multiplicity of infection of 10 was evaluated after $48 \mathrm{~h}$ treatment with siRNA-ERK1/2 or siRNA-NC (control group) (A) using fluorescence-assisted cell sorting analysis. Values are expressed as the mean of triplicate measurements \pm standard error. ${ }^{*} \mathrm{P}<0.05$ vs. NC. (B) The infectivity was also assessed using fluorescence microscopy (scale bar, $200 \mu \mathrm{m}$ ). Under the same conditions, the left panel shows transmitted light images and the right panels show green fluorescence. JNK, c-Jun N-terminal kinase; ERK, extracellular signal-regulated kinase; siRNA, small interfering RNA; NC, negative control; GFP, green fluorescence protein. CAR, coxsackie and adenovirus receptor; $\mathrm{mCSC}$, murine cardiac stem cell.

A
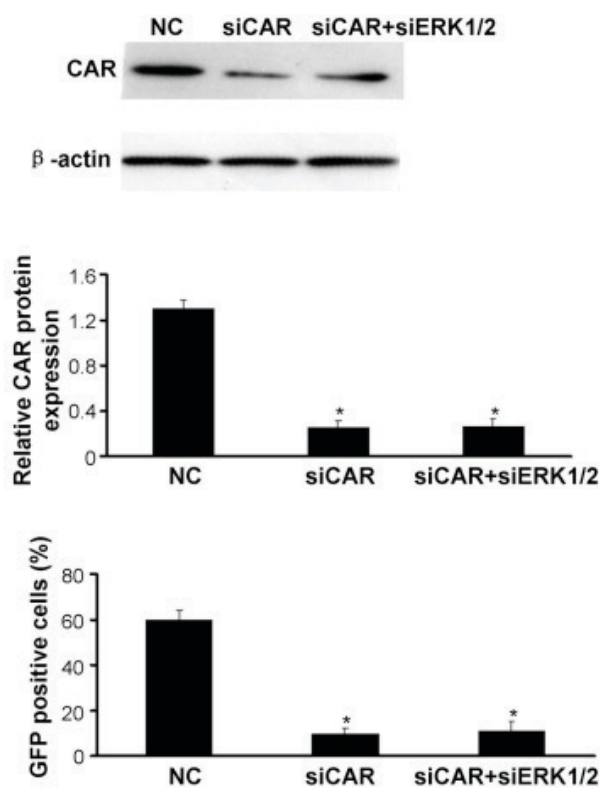

B

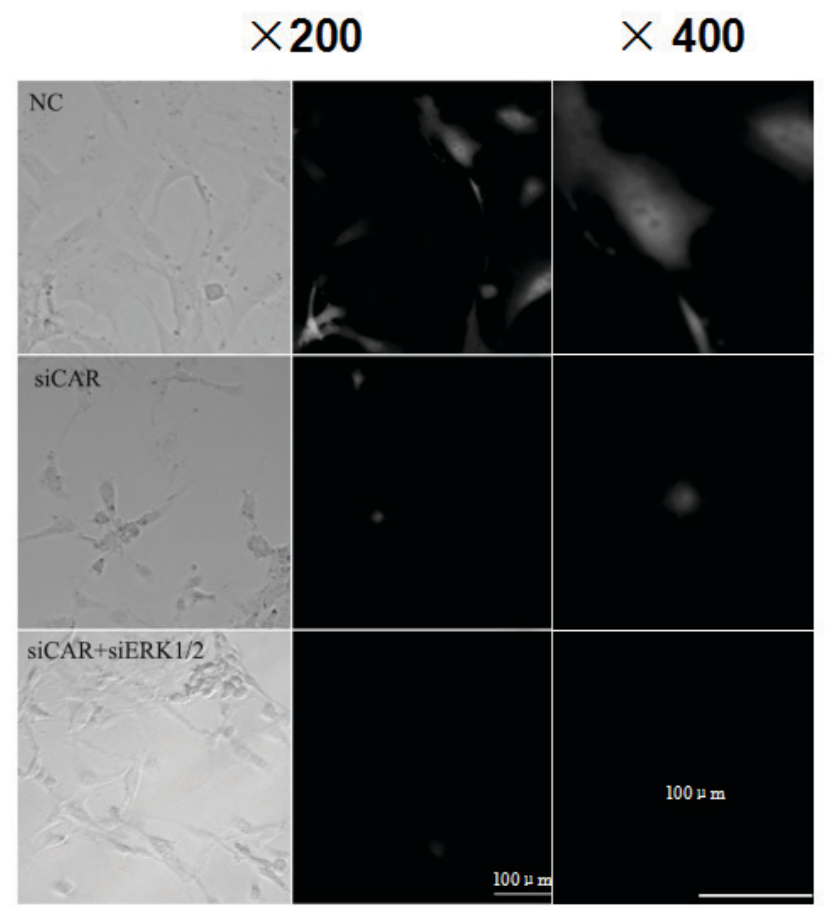

Figure 5. Changes in viral uptake and CAR expression after silencing of ERK1/2. mCSCs were treated with siRNA-CAR alone or siRNA-CAR with siRNA-ERK1/2 for $48 \mathrm{~h}$. siRNA-NC-treated mCSCs were used as a control group. (A) CAR protein expression was detected by immunoblot analysis and densitometrically quantified. The bar graphs represent the mean values of triplicate measurements \pm standard error of the mean. ${ }^{*} \mathrm{P}<0.05 \mathrm{vs}$. NC. (B) The infectivity of a GFP-expressing, non-replicating adenovirus at a multiplicity of infection of 10 was evaluated by fluorescence microscopy (scale bar, $100 \mu \mathrm{m}$ ). Under the same conditions, the left panel shows transmitted light images and the right panels show fluorescence. ERK, extracellular signal-regulated kinase; siRNA, small interfering RNA; NC, negative control; GFP, green fluorescence protein; CAR, coxsackie and adenovirus receptor; mCSC, murine cardiac stem cell. 
stem cells. To test this hypothesis, CSCs were treated with siRNA-ERK1/2, -JNK and -p38, and the effects on CAR expression and Ad uptake were assessed.

Treatment of CSCs with siRNA-ERK1/2 led to an increase in CAR protein levels at the cell membrane, with marked upregulation observed at sites of cell-cell contact. Relocation of CAR at the plasma membrane following the inhibition of ERK was sufficient to enhance the uptake of Ad. The aforementioned effects appear to depend on CAR specifically, as silencing of CAR significantly reduced Ad uptake, which could not be rescued by co-transfection with ERK1/2-siRNA. Furthermore, the effects of the other branches of the MAPK pathway were examined; however, neither the expression of CAR nor Ad uptake were affected by the silencing of JNK or P38. These results suggest that inhibition of Raf-MEK-ERK signaling may affect not only the expression of CAR, but also its sub-cellular localization on the membrane to improve the uptake of Ad by mCSCs.

\section{Acknowledgements}

The present study was funded by the Key Laboratory of Myocardial Ischemia Mechanism and Treatment (grant no. KF201304), the Heilongjiang Postdoctoral Foundation Assistance (grant no. LBH-Z14218) and the China Postdoctoral Science Foundation Grant (no. 2014M56137). The authors would like to thank Dr Zhaohua Zhong (Department of Microbiology, Harbin Medical University, Harbin, China) for their help.

\section{References}

1. Bergmann O, Bhardwaj RD, Bernard S, Zdunek S Barnabé-Heider F, Walsh S, Zupicich J, Alkass K, Buchholz BA and Druid H: Evidence for cardiomyocyte renewal in humans. Science 324: 98-102, 2009.

2. Hsieh PC, Segers VF, Davis ME, MacGillivray C, Gannon J, Molkentin JD, Robbins J and Lee RT: Evidence from a genetic fate-mapping study that stem cells refresh adult mammalian cardiomyocytes after injury. Nat Med 13: 970-974, 2007.

3. Ylä-Herttuala S and Martin JF: Cardiovascular gene therapy. Lancet 355: 213-222, 2000.

4. Schneider MD and French BA: The advent of adenovirus. Gene therapy for cardiovascular disease. Circulation 88: 1937-1942, 1993.

5. Nabel EG: Gene therapy for cardiovascular disease. Circulation 91: 541-548, 1995.

6. Kalka C and Baumgartner I: Gene and stem cell therapy in peripheral arterial occlusive disease. Vasc Med 13: 157-172, 2008

7. Ly H, Kawase Y, Yoneyama R and Hajjar RJ: Gene therapy in the treatment of heart failure. Physiology (Bethesda) 22: 81-96, 2007.

8. McCormick F: Cancer gene therapy: Fringe or cutting edge? Nat Rev Cancer 1: 130-141, 2001.

9. St George J: Gene therapy progress and prospects: Adenoviral vectors. Gene Ther 10: 1135-1141, 2003.

10. Cohen CJ, Gaetz J, Ohman T and Bergelson JM: Multiple regions within the coxsackievirus and adenovirus receptor cytoplasmic domain are required for basolateral sorting. J Biol Chem 276: 25392-25398, 2001.

11. Krasnykh V, Belousova N, Korokhov N, Mikheeva G and Curiel DT: Genetic targeting of an adenovirus vector via replacement of the fiber protein with the phage T4 fibritin. J Viro 75: 4176-4183, 2001.
12. Okegawa T, Pong RC, Li Y, Bergelson JM, Sagalowsky AI and Hsieh JT: The mechanism of the growth-inhibitory effect of coxsackie and adenovirus receptor (CAR) on human bladder cancer: A functional analysis of car protein structure. Cancer Res 61: 6592-6600, 2001.

13. Wickham TJ, Mathias P, Cheresh DA and Nemerow GR: Integrins alpha $\mathrm{v}$ beta 3 and alpha $\mathrm{v}$ beta 5 promote adenovirus internalization but not virus attachment. Cell 73: 309-319, 1993.

14. Li Y, Pong RC, Bergelson JM, Hall MC, Sagalowsky AI, Tseng CP, Wang Z and Hsieh JT: Loss of adenoviral receptor expression in human bladder cancer cells: A potential impact on the efficacy of gene therapy. Cancer Res 59: 325-330, 1999.

15. Okegawa T, Li Y, Pong RC, Bergelson JM, Zhou J and Hsieh JT: The dual impact of coxsackie and adenovirus receptor expression on human prostate cancer gene therapy. Cancer Res 60: 5031-5036, 2000.

16. Rauen KA, Sudilovsky D, Le JL, Chew KL, Hann B, Weinberg V, Schmitt LD and McCormick F: Expression of the coxsackie adenovirus receptor in normal prostate and in primary and metastatic prostate carcinoma: Potential relevance to gene therapy. Cancer Res 62: 3812-3818, 2002.

17. Chen Yh, Lu Q, Schneeberger EE and Goodenough DA: Restoration of tight junction structure and barrier function by down-regulation of the mitogen-activated protein kinase pathway in ras-transformed Madin-Darby canine kidney cells. Mol Biol Cell 11: 849-862, 2000.

18. Hemminki A, Kanerva A, Liu B, Wang M, Alvarez RD, Siegal GP and Curiel DT: Modulation of coxsackie-adenovirus receptor expression for increased adenoviral transgene expression. Cancer Res 63: 847-853, 2003

19. Farmer C, Morton PE, Snippe M, Santis G and Parsons M: Coxsackie adenovirus receptor (CAR) regulates integrin function through activation of p44/42 MAPK. Exp Cell Res 315: 2637-2647, 2009.

20. Beltrami AP, Barlucchi L, Torella D, Baker M, Limana F, Chimenti S, Kasahara H, Rota M, Musso E and Urbanek K: Adult cardiac stem cells are multipotent and support myocardial regeneration. Cell 114: 763-776, 2003.

21. Liu J, Wang Y, Du W, Liu W, Liu F, Zhang L, Zhang M, Hou M, Liu K, Zhang S and Yu B: Wnt1 inhibits hydrogen peroxide-induced apoptosis in mouse cardiac stem cells. PloS One 8: e58883, 2013.

22. Liu J, Wang Y, Du W and Yu B: Sca-1-positive cardiac stem cell migration in a cardiac infarction model. Inflammation 36 : 738-749, 2013.

23. Wang Y, Liu J, Cui J, Sun M, Du W, Chen T, Ming X, Zhang L, Tian J, Li J, et al: MiR218 modulates wnt signaling in mouse cardiac stem cells by promoting Proliferation and Inhibiting Differentiation through a Positive Feedback Loop. Sci Rep 6: 20968, 2016.

24. Livak KJ and Schmittgen TD: Analysis of relative gene expression data using real-time quantitative PCR and the 2(-Delta Delta C(T)). Methods 25: 402-408, 2001.

25. Bergelson JM, Cunningham JA, Droguett G, Kurt-Jones EA, Krithivas A, Hong JS, Horwitz MS, Crowell RL and Finberg RW: Isolation of a common receptor for Coxsackie B viruses and adenoviruses 2 and 5. Science 275: 1320-1323, 1997.

26. van Raaij MJ, Chouin E, van der Zandt H, Bergelson JM and Cusack S: Dimeric structure of the coxsackievirus and adenovirus receptor D1 domain at $1.7 \AA$ resolution. Structure 8: $1147-1155,2000$

27. Honda T, Saitoh H, Masuko M, Katagiri-Abe T, Tominaga K, Kozakai I, Kobayashi K, Kumanishi T, Watanabe YG, Odani S and Kuwano R: The coxsackievirus-adenovirus receptor protein as a cell adhesion molecule in the developing mouse brain. Brain Res Mol Brain Res 77: 19-28, 2000.

28. Walters RW, Freimuth P, Moninger TO, Ganske I, Zabner J and Welsh MJ: Adenovirus fiber disrupts CAR-mediated intercellular adhesion allowing virus escape. Cell 110: 789-799, 2002. 